\title{
Thrush, CTCAE
}

National Cancer Institute

\section{Source}

National Cancer Institute. Thrush, CT CAE. NCI Thesaurus. Code C143214.

A disorder characterized by a suspected candidal infection involving an oral mucosal surface. 